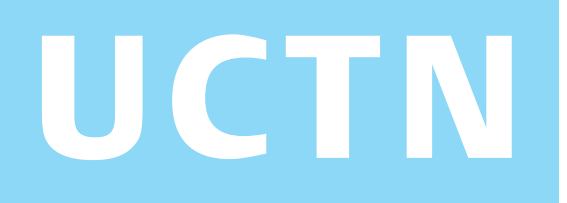

\title{
Endoscopic submucosal dissection using a novel irrigation wiper-knife
}
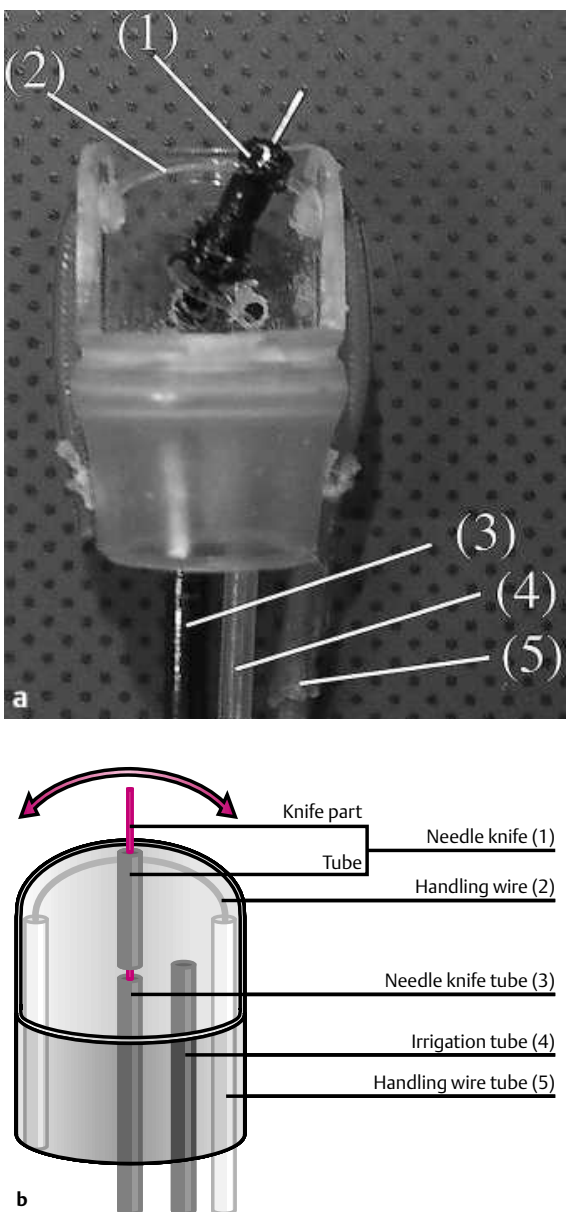

Figure 1 The irrigation wiper-knife. a The irrigation tube (4) and needle-knife (1) were glued to the exterior surface over the hole, but only the needle-knife was intersected by and fixed with the handling wire (2). b The schematic representation shows more clearly how the needle-knife moves like a windshield wiper (double-headed red arrow).

Endoscopic submucosal dissection techniques require special skills and the procedure can be very prolonged $[1,2]$. In response these drawbacks, we have designed a new dissection knife, the "wiper-knife", which simplifies the procedure.

We recently developed a hood-knife that facilitates endoscopic submucosal dissection by sliding the hood-knife with coagulation current along the muscle layer [2]. However, we considered that it was essential to be able to perform endoscopic

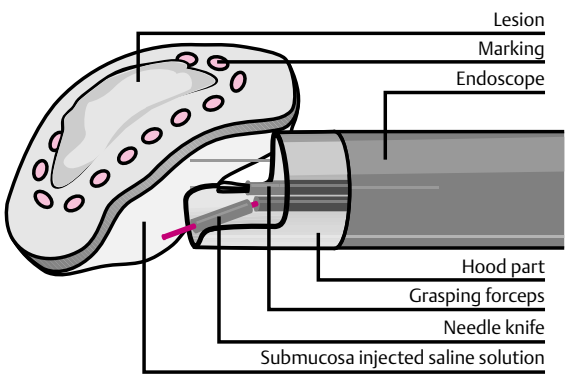

Figure 2 Schematic representation of the endoscopic submucosal dissection procedure using the wiper-knife.

submucosal dissection more mechanically. The wiper-knife was made by installing a needle-knife in exchange for the snare forceps we used in the hood-knife. The needle-knife was crossed by and fixed with a handling wire, which was inserted through tubes at either side of the hood.

The endoscopic submucosal dissection procedure using the wiper-knife is carried out as follows (Figure 2): a grasping forceps is passed through an accessory channel and used to push the lesion away from the muscle layer; submucosal exfoliation is then performed by moving the wiperknife like a windshield wiper with coagulation current on the muscle layer to separate the submucosa from the muscle layer. We resected three specimens in animal models (pigs) using this wiper-knife. The average diameter of the resected specimens was $30 \mathrm{~mm}$.

Endoscopic submucosal dissection can be safely and easily carried out under direct vision using this method. Another advantage of the wiper-knife is that endoscopic submucosal dissection and endoscopic hemostasis can be carried out while simultaneously applying adequate irrigation [2-6].

Endoscopy_UCTN_Code_TTT_1AO_2AG

\section{K. Kume, M. Yamasaki, K. Kanda, I. Yoshikawa, M. Otsuki}

Third Department of Internal Medicine, School of Medicine, University of Occupational and Environmental Health, Kitakyusyu, Japan.

\section{References}

${ }^{1}$ Muto M, Miyamoto S, Hosokawa A et al. Endoscopic mucosal resection in the stomach using the insulated-tip needle-knife. Endoscopy 2005; 37: $178-182$

${ }^{2}$ Kume K, Yamasaki M, Kanda K et al. Endoscopic submucosal dissection using a novel irrigation hood-knife. Endoscopy 2005; 37: $1030-1031$

${ }^{3}$ Kume K, Yamasaki M, Yamasaki T et al. Endoscopic hemostatic treatment under irrigation for upper GI hemorrhage: a comparison of one third and total circumference transparent end hoods. Gastrointest Endosc 2004; 59: 712 - 716

${ }^{4}$ Kume K, Yoshikawa I, Otsuki M. Endoscopic treatment of upper GI hemorrhage with a novel irrigating hood attached to the endoscope. Gastrointest Endosc 2003; 57: $732-$ 735

${ }^{5}$ Kume K, Yamasaki M, Kubo K et al. EMR of upper GI lesions when using a novel soft, irrigation, prelooped hood. Gastrointest Endosc 2004; 60: 124-128

${ }^{6}$ Kume K, Yamasaki M, Kanda K et al. Endoscopic procedure under irrigation. Dig Endosc 2005; 17: $241-245$

\section{Corresponding author}

\section{Otsuki, M.D.}

Third Department of Internal Medicine School of Medicine

University of Occupational and Environmental Health, 1-1 Iseigaoka, Yahatanishi-ku, Kitakyusyu 807-8555 Japan

Fax: $\quad+81-93-692-0107$

Email: mac-otsk@med.uoeh-u.ac.jp 